Noname manuscript No.

(will be inserted by the editor)

\title{
Shot noise suppression due to a magnetic field in disordered conductors
}

\author{
M. Macucci · P. Marconcini
}

Received: date / Accepted: date

\begin{abstract}
We investigate the effect of an orthogonal magnetic field on a 2-D disordered wire, by means of a numerical model based on the recursive Green's function technique. We discuss the resulting behavior of the shot noise suppression factor and of the conductance in terms of the interplay among the relevant transport quantities, i.e. the mean free path, the localization length, the average separation between impurities and the cyclotron radius. We find that, starting from a diffusive or quasi-diffusive behavior, shot noise is increasingly suppressed as the magnetic field is turned on, up to a noiseless condition typical of the disappearance of backscattering for edge states.
\end{abstract}

\section{Introduction}

Shot noise suppression in ballistic and diffusive conductors has been the subject of an intense research effort in the last two decades [1], with quite interesting results from both the theoretical and the experimental point of view.

In particular, it is well-known that the shot noise power spectral density is suppressed in disordered conductors with respect to the full shot noise power spectral density yielded by Schottky's theorem [2]. Beenakker and Büttiker [3] predicted, based on random matrix theory [4], that shot noise in a diffusive conductor is reduced down to $1 / 3$ of its full value. This effect was experimentally confirmed by Henny et al. [5] for metallic diffusive wires, while its observation

M. Macucci and P. Marconcini

Dipartimento di Ingegneria dell'Informazione

Università di Pisa

via Girolamo Caruso 16

I-156122 PISA, Italy

Tel.: +39050 2217537

Fax: +39050 2217522

E-mail: macucci@mercurio.iet.unipi.it has proved to be elusive in semiconductors [6], mainly because of the difficulty in achieving the right parameter combination in a semiconductor nanostructure [7]. A few years ago we observed [8] that application of an orthogonal magnetic field led to further suppression of shot noise below the $1 / 3$ value, but at the time we did not investigate the issue in detail, mainly due to the computational cost of an in-depth analysis of this effect. Here we report the results of numerical simulations made possible by the availability of better computational resources and of improved codes, aimed at obtaining a more accurate picture of the dependence of shot noise suppression on magnetic field. We will focus on the role played by an orthogonal magnetic field on shot noise suppression in two dimensional disordered conductors, analyzing the behavior in terms of the conductance, the localization length, the cyclotron radius and the average separation between impurities, which we assume as hard-wall scatterers, since we have previously observed [7,9] that their effect on shot noise suppression is substantially equivalent to that of a more realistic but more computationally expensive softwall disordered potential.

The paper is organized as follows: in the next section we discuss the numerical method that we have adopted, based on the recursive Green's function approach [10-13], while in the third section we report the results of the numerical simulation and their interpretation.

\section{Numerical Method}

We have evaluated the conductance and the shot noise power spectral density from the transmission matrix of the structure, which has been computed with the recursive Green's function method.

Defining $x, y$ and $z$ as the coordinates in the longitudinal, transverse and orthogonal directions (and $\hat{\mathbf{x}}, \hat{\mathbf{y}}$ and $\hat{\mathbf{z}}$ as 
the corresponding unit vectors), we include the effect of a magnetic field $\mathbf{B}=B \hat{\mathbf{z}}$ orthogonal to the plane containing the device by means of a Landau gauge with a transversallyoriented vector potential $\mathbf{A}=(B x) \hat{\mathbf{y}}$.

In our simulations we subdivide the structure into a series of cascaded sections, within each of which both the electrostatic and the vector potential can be approximated as longitudinally constant.

With the adopted Landau gauge, in each section the solutions of the Schrödinger equation coincide with those in the absence of a magnetic field, except for the multiplication of the transverse component of the wave function by a Peierls phase factor [14-16]. In detail, if in the $i$-th section (with longitudinal coordinate $x_{i}$ ) the $n$-th transport mode, in the absence of a magnetic field, is given by

$\psi_{n, x_{i}}^{0}(x, y)=\chi_{n, x_{i}}^{0}(y) e^{i k_{n, x_{i}}^{0} x}$,

in the presence of a magnetic field it becomes

$\psi_{n, x_{i}}(x, y)=\left[\chi_{n, x_{i}}^{0}(y) e^{-i \frac{e}{\hbar} B x_{i} y}\right] e^{i k_{n, x_{i}}^{0} x}$,

where $\chi(y)$ is the transverse component of the wave function, $k$ the longitudinal wave vector, $e$ the elementary charge, and $\hbar$ the reduced Planck constant.

We use a mixed representation for the Green's functions [11-13]: in the real space along $x$ and in the space of the transverse eigenmodes along $y$. Therefore, the Green's function $G$ relates two points along $x$ and two modes along $y$. For the numerical simulations, a grid of discretization nodes with a step $a$ is used along $x$; the nodes will be identified with their position number.

We first assume the sections as isolated from one another, with Dirichlet boundary conditions at their ends. In this case, the Green's function matrix between any pair of points along $x$ (which is a function of the transverse modes) can be represented with a diagonal matrix because no modemixing exists inside each longitudinally-invariant section. As we will detail below, for each element of this matrix, which represents an independent one-dimensional problem, an analytical expression is available, as a function of the longitudinal wave vector $k$ and discretization step $a$.

Once the Green's functions of the single isolated sections have been computed, they can be recursively composed using the Dyson equation.

Let us consider a couple of adjacent sections and let us call $V$ the part of Hamiltonian (written on the basis of the nodes along $x$ and of the modes along $y$ ) which couples the two sections (if $H^{0}$ and $H$ are the Hamiltonians of the system when the sections are uncoupled and coupled, respectively, then $\left.H=H^{0}+V\right)$. If $G_{0}(G)$ is the Green's function of the unperturbed (perturbed) system represented by the two uncoupled (coupled) sections, then:

$G=G^{0}+G^{0} V G$

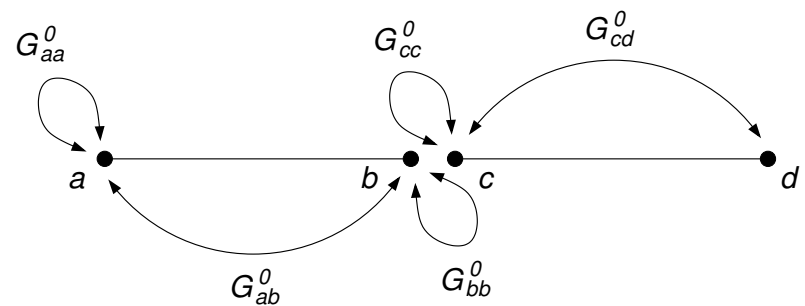

Fig. 1 Sketch of the Green's functions required for the composition of two adjacent section, extending from the node $a$ to the node $b$, and from the node $c$ to the node $d$, respectively.

(Dyson equation). This is an implicit equation, but it can be recast into a set of explicit relations.

In detail, let us define $G_{i j}=\langle i|G| j\rangle$ the Green's function between the generic nodes $i$ and $j$ of the discretization grid along the $x$ direction. When composing the section running from node $a$ to node $b$ with the section running from node $c$ (adjacent to $b$ ) to node $d, G_{a d}$ and $G_{a a}$ can be computed from the Green's functions represented in Fig. 1 using the following relations:

$G_{a a}=G_{a a}^{0}+G_{a b}^{0} V_{b c}\left(I-G_{c c}^{0} V_{c b} G_{b b}^{0} V_{b c}\right)^{-1} G_{c c}^{0} V_{c b} G_{b a}^{0}$

$G_{a d}=G_{a b}^{0} V_{b c}\left(I-G_{c c}^{0} V_{c b} G_{b b}^{0} V_{b c}\right)^{-1} G_{c d}^{0}$,

which derive from Eq. (3).

Note that since the term which, in the real space, couples two adjacent nodes along $x$ is $v=-\hbar^{2} /\left(2 m a^{2}\right)$ (where $m$ is the effective mass of the electrons), the elements of the matrix $V_{i j}$ coupling adjacent nodes $i$ and $j$ of different sections are equal to the overlap integrals of the transverse modes in the two neighboring sections multiplied by $v$.

The relations (4) are used as follows. We assume two semi-infinite leads attached to the left and right ends of the device, in order to enforce absorbing boundary conditions. Starting from the right semi-infinite lead, for which, as we will shortly detail, we know $G_{c c}^{0}$ and $G_{c d}^{0}$, and from the adjacent section at its left, for which $G_{a a}^{0}, G_{a b}^{0}$ and $G_{b b}^{0}$ are available, we compute the Green's functions $G_{a a}$ and $G_{a d}$ of the block deriving from their composition. Considering these two functions as the new $G_{c c}^{0}$ and $G_{c d}^{0}$, we can further compose this block with the section at its left, again using Eqs. (4). Recursively repeating this procedure for all the sections up to the left semi-infinite lead, we can obtain the Green's functions of the overall device.

As we have briefly mentioned, the Green's functions, between pairs of nodes, of the semi-infinite leads and of the single sections (which we have exploited in the described procedure) have a diagonal matrix representation in the space of the transverse modes. The analytical expressions of the elements of these matrices, one for each mode (let us define $\left(G_{i j}^{0}\right)_{n}=\left\langle n\left|G_{i j}^{0}\right| n\right\rangle$ for a generic mode $n$ ), can be derived exploiting the expression of the Green's function between two generic nodes of an infinite one-dimensional discretized chain $[17,18]$ and are reported in the following. 
For the semi-infinite right lead, with first node $c$ and a generic node $d$ :

$\left(G_{c d}^{0}\right)_{n}=\frac{e^{i N \theta_{n}}}{v}, \quad\left(G_{c c}^{0}\right)_{n}=\frac{e^{i \theta_{n}}}{v}$,

where $\theta_{n}=k_{n} a$ (with $k_{n}$ the longitudinal wave vector for the mode $n$ ) and $N=|d-c|+1$ (the number of nodes from $c$ to d).

For each single finite section, extending from the node $a$ to the node $b$ :

$$
\begin{gathered}
\left(G_{a b}^{0}\right)_{n}=\frac{\sin \theta_{n}}{v \sin (N+1) \theta_{n}}, \\
\left(G_{a a}^{0}\right)_{n}=\left(G_{b b}^{0}\right)_{n}=\frac{\sin N \theta_{n}}{v \sin (N+1) \theta_{n}},
\end{gathered}
$$

where $N=|b-a|+1$ (the number of nodes from $a$ to $b$ ).

Finally, for the semi-infinite left lead, with generic node $a$ and last node $b$ :

$$
\begin{gathered}
\left(G_{a b}^{0}\right)_{n}=\frac{e^{i N \theta_{n}}}{v}, \\
\left(G_{a a}^{0}\right)_{n}=\frac{e^{i N \theta_{n}}}{v} \frac{\sin N \theta_{n}}{\sin \theta_{n}}, \quad\left(G_{b b}^{0}\right)_{n}=\frac{e^{i \theta_{n}}}{v},
\end{gathered}
$$

where $N=|b-a|+1$ (the number of nodes from $a$ to $b$ ).

Once the Green's functions of the overall device have been computed, by means of the recursive procedure we have described, it is possible to obtain the transmission and reflection matrices. The elements of the transmission matrix between the nodes $j$ and $l$ and of the reflection matrix at the node $l$ are given by $[11,19]$

$$
\begin{aligned}
t_{n m}=-i 2 v\left(\sin \theta_{n} \sin \theta_{m}\right)^{1 / 2} e^{i\left(\theta_{m} l-\theta_{n} j\right)}\left\langle n\left|G_{j l}\right| m\right\rangle, \\
r_{n m}=-\left(\sin \theta_{n} / \sin \theta_{m}\right)^{1 / 2} e^{i 2\left(\theta_{n}+\theta_{m}\right) l} \times \\
\left(i 2 v \sin \theta_{m}\left\langle n\left|G_{l l}\right| m\right\rangle+\delta_{m n}\right),
\end{aligned}
$$

where $n$ and $m$ specify two propagating modes (at the nodes $j$ and $l$, respectively, in Eq. (8); both at the node $l$ in Eq. (9)) and $\delta_{m n}$ is a Kronecker delta.

In order to exploit the relations (8)-(9), which are valid in the absence of magnetic field, we have considered a magnetic field which is null at the nodes of the leads between which the Green's functions are computed and then linearly increases (from the leads towards the center of the structure) until it reaches the desired constant value, which is then kept constant across the operating region of the device (see Fig. 2).

The length of the sections into which the device is subdivided is limited by the fact that the method is valid only if the longitudinal variations of the electrostatic and the vector potential within each section are negligible. In particular, the flux of the magnetic field through each section has to be less than the magnetic flux quantum $h /(2 e)$ [14]. For high values of the magnetic field and for wide structures this constraint strongly increases the number of sections that have to be considered and thus the computational times.

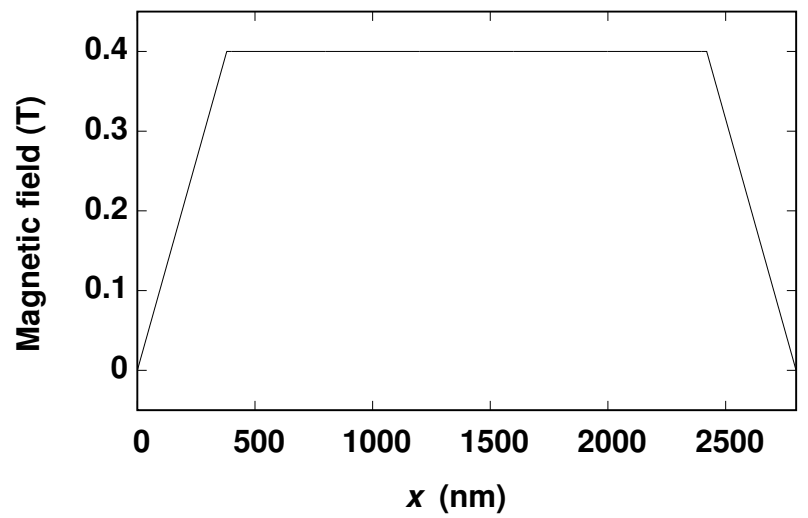

Fig. 2 Value of the orthogonal magnetic field as a function of the longitudinal coordinate; the magnetic field is ramped from zero to its full value (constant in the active region) and than back to zero.

Once the transmission matrix of the overall device is obtained, its conductance $G$ and the shot noise power spectral density $S_{I}$ can be computed exploiting the LandauerBüttiker approach [20,21]. In particular, if $w_{i}$ are the eigenvalues of the matrix $t^{\dagger} t, G$ and $S_{I}$ can be obtained from:

$G=\frac{2 e^{2}}{h} \sum_{i} w_{i}, S_{I}=\frac{4 e^{2}}{h}|e V| \sum_{i} w_{i}\left(1-w_{i}\right)$,

where $V$ is the voltage applied between the contacts of the device. As a consequence, the Fano factor $F$ (i.e., the shot noise suppression factor with respect to the full shot noise power spectral density $\left.S_{I}=2 e|I|=2 e|V| G\right)$ is given by

$F=\frac{\left\langle\sum_{j} w_{i}\left(1-w_{i}\right)\right\rangle}{\left\langle\sum_{i} w_{i}\right\rangle}$

where the angle brackets indicate an average over the electron energies.

Notice that the choice of the exact positions in the leads of the points between which the Green's function is computed has no influence on $G, S_{I}$ and $F$ because, as it is apparent in Eq. (8), it modifies only the phase of the transmission matrix.

Since here we are interested in the evaluation of shot noise, we assume a bias condition for which shot noise is prevalent with respect to thermal noise and thus we consider an applied voltage $V$ between the terminals much greater than $k T / e$ (where $k$ is the Boltzmann constant and $T$ is the absolute temperature). In these conditions, on an energy range $\mathrm{eV}$ the Fermi-Dirac distribution function can be approximated with a step function and thus the averages in Eq. (11) are uniform averages over all the energies between the Fermi levels of the two contacts. These averages have to be performed separately for the numerator and the denominator (as in actual measurements, where noise and current are measured separately), before taking the ratio.

Since, especially in disordered samples with a low number of randomly located scatterers, the results strongly depend on their actual distribution, sometimes it can also be 


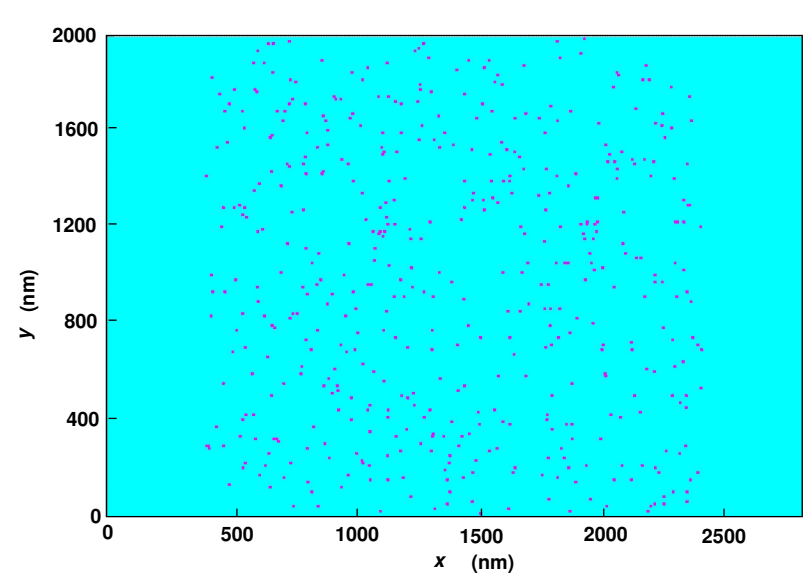

Fig. 3 Realization of the disordered potential, with 500 square $10 \mathrm{~nm}$ $\times 10 \mathrm{~nm}$ obstacles, with a height of $40 \mathrm{meV}$.

useful to perform an average of the values of Fano factor obtained for several scatterer distributions. From an experimental point of view, this corresponds to averaging the results obtained measuring different samples, in order to obtain an estimate of the most likely conductance and noise values.

\section{Results and Discussion}

The first structure that we have studied is a square 2-D conductor with a size of $2 \mu \mathrm{m} \times 2 \mu \mathrm{m}$ and 500 hard-wall square $10 \mathrm{~nm} \times 10 \mathrm{~nm}$ randomly distributed scatterers. The Fermi energy is assumed to be $6.24 \mathrm{meV}$ (as in all the following simulations), and results are averaged over 21 uniformly spaced energy values in a range of $40 \mu \mathrm{eV}$ around the Fermi level, which corresponds to $\pm 10 k T$, for $T \simeq 23 \mathrm{mK}$. A realization of the scatterer landscape is reported in Fig 3.

The density of scatterers is chosen in such a way that transport is in the diffusive regime, i.e. the inequality $l_{e l} \ll$ $L \ll l_{l o c}$ is satisfied, where $l_{e l}$ is the elastic mean free path, $L$ is the device length and $l_{l o c}$ is the localization length, which, in the presence of 2-D or 3-D disorder is about $N l_{e l}$ in the absence of a magnetic field and about twice such value in the presence of a magnetic field [7,4,22,23].

In Fig. 4 we show the computed conductance, in units of the conductance quantum $G_{0}=2 e^{2} / h$ (where $h$ is the Planck constant), as a function of magnetic field. There is a slight increase of the conductance as the magnetic field is turned on (probably due to the suppression of weak localization) and then a substantially linear decrease.

Indeed for zero or very small magnetic field the Fano factor (see Fig. 5) behaves as expected for a diffusive conductor, with a value around $1 / 3$. It is to be noticed that the range of Fermi energies for which, for the chosen device geometry and scatterer density, a diffusive behavior is kept, is rather limited, as we have discussed in general in Ref. [7].

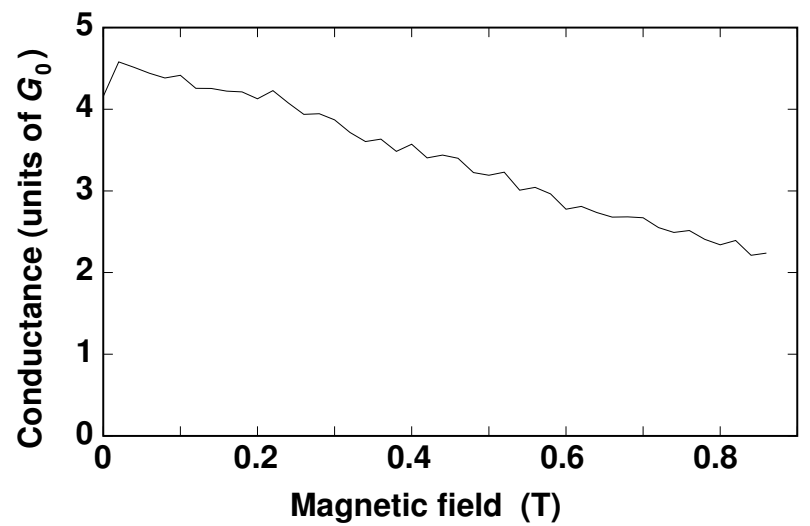

Fig. 4 Conductance as a function of the magnetic field for a diffusive $2 \mu \mathrm{m} \times 2 \mu \mathrm{m}$ conductor.

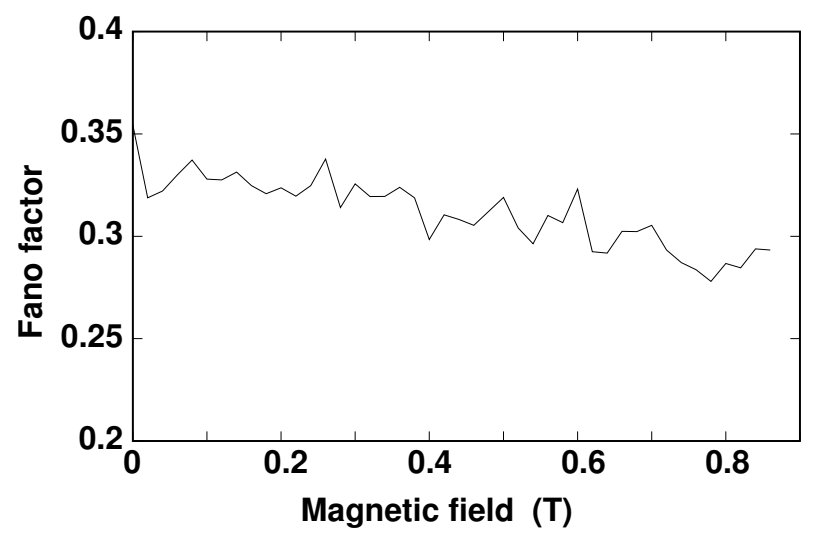

Fig. 5 Fano factor as a function of the magnetic field for a diffusive $2 \mu \mathrm{m} \times 2 \mu \mathrm{m}$ conductor.

As the magnetic field is increased, we observe a substantially linear decrease of the Fano factor. It is interesting to understand the origin of this decrease, and, in particular, whether it is associated with a departure from the diffusive limit due to a reduction of the number of propagating modes or to suppressed scattering. The former hypothesis would be based on the observation that, as the magnetic field confines the wave function in a portion of the width of wire, the number of propagating modes is reduced, which involves a reduction of the localization length (which we have previously seen to be proportional to the number of propagating interacting modes) and therefore makes it more difficult to satisfy the inequality $l_{e l} \ll L \ll l_{l o c}$. Indeed, looking at the Fano factor of narrower wires with no magnetic field a similar shot noise reduction is observed. The latter hypothesis, instead, stems from the consideration that, from a semiclassical point of view, the magnetic field bends the trajectories of the charge carriers into cyclotron orbits, thereby making it more difficult to backscatter or even completely suppressing backscattering when narrow edge states are formed [24]. 


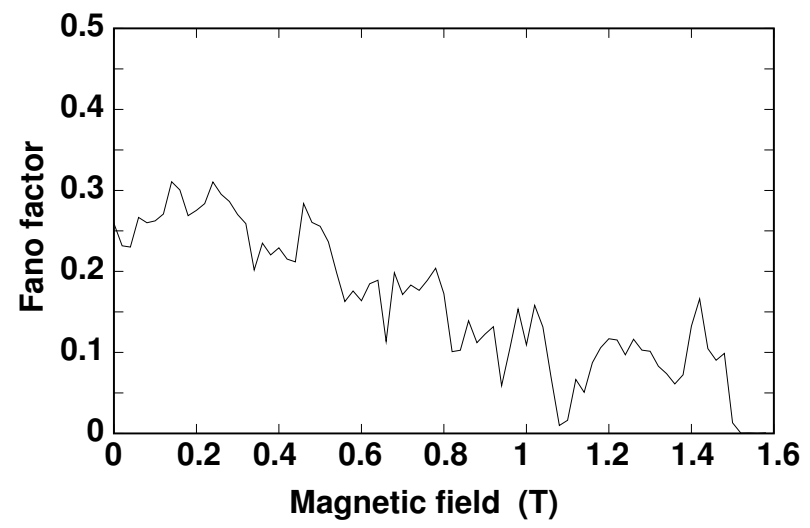

Fig. 6 Fano factor as a function of the magnetic field for a disordered $400 \mathrm{~nm} \times 600 \mathrm{~nm}$ conductor.

This is also a simple and intuitive way to explain the quantum Hall effect [24].

In order to decide between these two possible explanations, we have looked at the transmission eigenvalues, noticing that, as the magnetic field is increased, the number of significantly nonzero eigenvalues decreases, but their value is, on average, closer to 1 . This means that the second explanation, i.e. the progressive suppression of backscattering is at the root of the observed noise behavior.

In the following, we report the eigenvalue data for a smaller disordered structure, for which it is possible to perform calculations at higher values of the magnetic field within a reasonable amount of time.

Indeed, we have to satisfy the condition that each of the slices into which the structure under investigation is subdivided must be threaded by a flux less than the flux quantum. For the $2 \mu \mathrm{m} \times 2 \mu \mathrm{m}$ structure the number of slices needed is already around 4000 for values of the magnetic field of $0.8 \mathrm{~T}$.

The smaller structure (400 nm wide and $600 \mathrm{~nm}$ long), although not precisely in the diffusive regime for $B=0$, allows a much more detailed exploration of the parameter space. In particular, we are interested in reaching values of the magnetic field for which the cyclotron radius is smaller than the average separation between impurities, so that, according to Büttiker [24], backscattering should be completely suppressed. We have included 30 impurities, thus keeping their concentration the same as the one in the previous structure. Thus also in the new structure the average separation between scatterers is given by $l_{i m p}=\sqrt{(W L) / N_{\text {imp }}}=89.44$ $\mathrm{nm}$, where $W$ is the device width and $N_{i m p}$ is the number of scatterers.

In order to obtain a cyclotron radius of about $45 \mathrm{~nm}$ in Gallium Arsenide (the material whose parameters have been used for all the calculations), we need a magnetic field of about $1.6 \mathrm{~T}$, for which we can easily perform numerical simulations in a structure $400 \mathrm{~nm}$ wide.

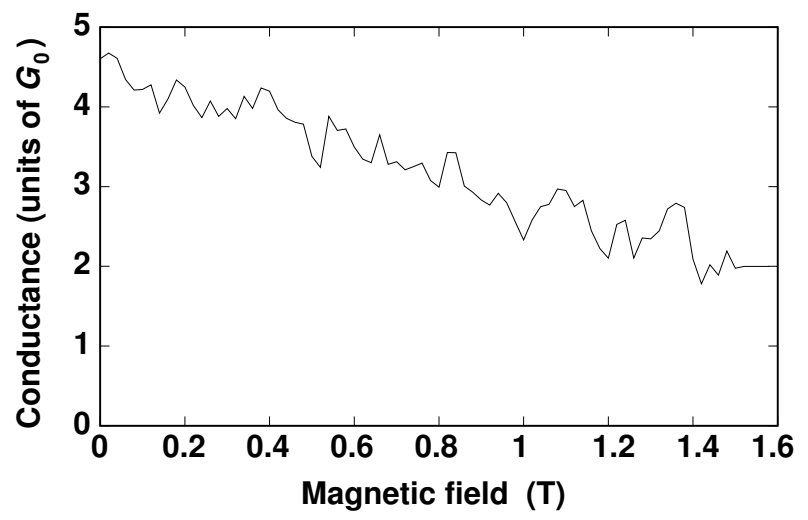

Fig. 7 Conductance as a function of the magnetic field for a $400 \mathrm{~nm} \times 600 \mathrm{~nm}$ conductor.

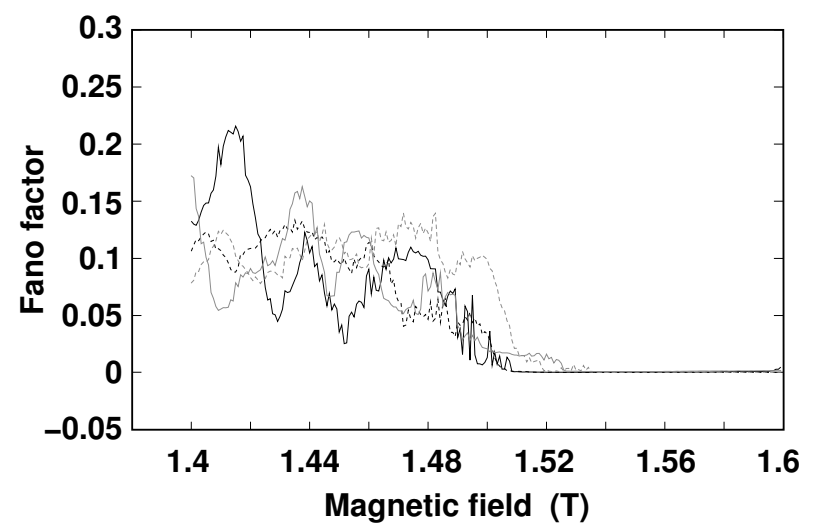

Fig. 8 Fano factor for a disordered conductor as a function of the magnetic field, in a narrow interval around the value for which noiseless edge states form.

The behavior of the Fano factor is reported in Fig. 6, where it is possible to see that there is a substantially linear decrease until, upon formation of the edge states without backscattering, the noise suddenly drops to zero, which is consistent with the behavior of the transmission eigenvalues. The behavior of the conductance is reported in Fig. 7.

We report also a close-up view (Fig. 8) of the Fano factor at the onset of the free-propagating edge states, for a few different distributions of the scatterers. We notice that the transition is rather sharp and occurs in a relatively small range of values of the magnetic field $(1.505 \mathrm{~T}<B<1.525 \mathrm{~T})$, for all the considered distributions. As soon as the ballistic edge states are formed, transmission for these two states is unitary and noise drops to zero.

In Fig. 9, we report the dependence of the largest 7 eigenvalues (the ones above 0.1 at $B=0$ ) on magnetic field. As the magnetic field is increased, the smaller eigenvalues drop to zero (due to magnetic confinement), while the two top ones, although with some fluctuations, get closer and closer to 1 , as a result of the formation of edge states free from backscattering. The data reported in Fig. 9 have been ob- 


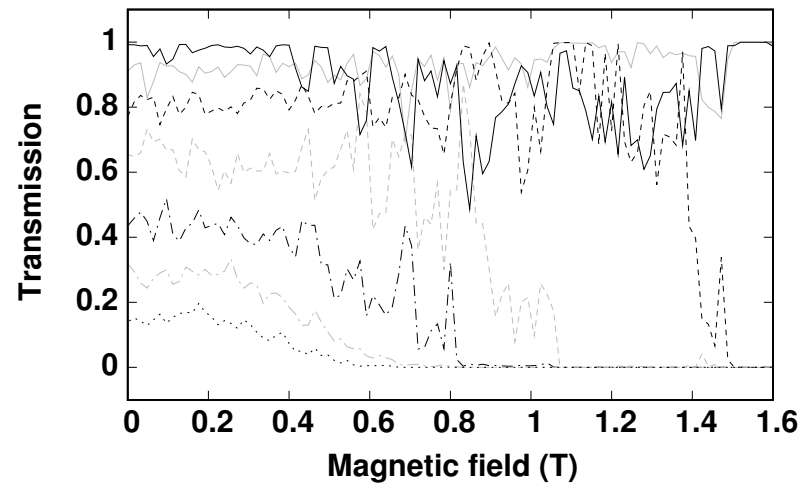

Fig. 9 Representation of the 7 largest eigenvalues, as a function of the magnetic field.

tained averaging over 6 different realizations of the scattering potential (using different seeds for the generation of the position of the hard-wall scatterers). We notice that the thresholds, in terms of magnetic field, for propagation of the eigenvalues are rather close to those for Landau levels in an unconfined 2-DEG (2-Dimensional Electron Gas), due to the relatively large width of the considered sample.

It is interesting to observe how the conductance and Fano factor behavior depend on the length of the device. To this purpose, we have repeated the calculations for structures that are twice and three times as long with respect to the one just described, while sharing all other parameters. Therefore the new structures have the same $400 \mathrm{~nm}$ width, but lengths of 1200 and $1800 \mathrm{~nm}$. In Fig. 10 we report a comparison of the conductance for the three structures: the conductance at $B=0$ is not exactly inversely proportional to the length, since, due to the limited width, we are not fully in the diffusive limit. However we are reasonably close to an ohmic condition, and it is interesting to examine the peculiar behavior of the conductance for the $1800 \mathrm{~nm}$ long device. In this case there is no substantial variation as a function of the magnetic field. Examining the transmission eigenvalues, we see that for $B=0$ there are 5 eigenvalues with a nonnegligible value: $0.9095,0.6008,0.2417,0.09235,0.02632$; as the magnetic field is increased, they undergo an initial increase (that can be interpreted as the suppression of weak localization) and then fluctuate, with a sharp drop in the value of the smaller ones. At about $0.8 \mathrm{~T}$ there are only three eigenvalues left that contribute significantly to the overall transmission: $0.8675,0.6803,0.1001$. Further increasing the magnetic field up to $1.6 \mathrm{~T}$, only two eigenvalues are left, both approximately unitary.

On the other hand, the Fano factor (see Fig. 11) has a behavior that is very similar for all lengths, with a linear decrease as the magnetic field is increased, and then a sharp drop down to zero around $1.5 \mathrm{~T}$, when the cyclotron radius becomes less than half of the average separation between impurities.

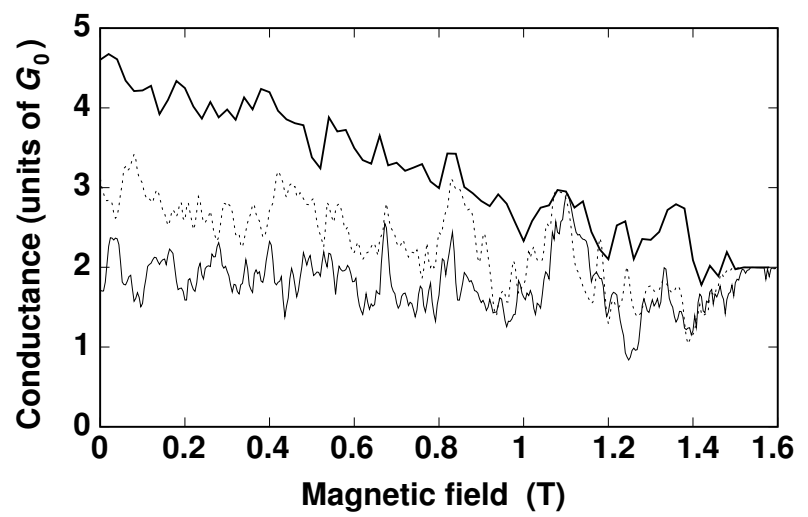

Fig. 10 Conductance comparison, as a function of magnetic field, for disordered conductors $400 \mathrm{~nm}$ wide and with a length of $600 \mathrm{~nm}$ (thick solid line), $1200 \mathrm{~nm}$ (dashed line), and $1800 \mathrm{~nm}$ (thin solid line).

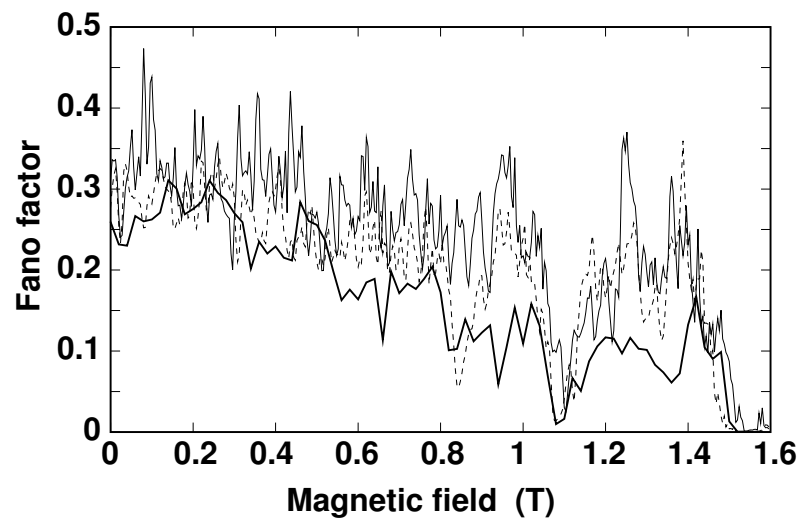

Fig. 11 Comparison of the Fano factor, as a function of magnetic field, for disordered conductors $400 \mathrm{~nm}$ wide and with a length of $600 \mathrm{~nm}$ (thick solid line), $1200 \mathrm{~nm}$ (dashed line), and $1800 \mathrm{~nm}$ (thin solid line).

Therefore we can conclude that in 2-dimensional disordered conductors an orthogonal magnetic field affects the noise behavior mainly through the reduction of backscattering (contrary to the case of 1-D disorder, where the main action is through the increase of the localization length) and the resulting "regularization" of the flow of electrons.

\section{References}

1. Ya. M. Blanter, M. Büttiker, "Shot noise in mesoscopic conductors," Physics Reports 336, 1 (2000).

2. W. Schottky, "Über spontane Stromschwankungen in verschiedenen Elektrizitätsleitern,” Ann. Phys. (Leipzig, Ger.) 57, 541 (1918).

3. C. W. J. Beenakker, M. Büttiker "Suppression of shot noise in metallic diffusive conductors," Phys. Rev. B 46, 1889(R) (1992).

4. C. W. J. Beenakker, "Random-matrix theory of quantum transport," Rev. Mod. Phys. 69, 731 (1997).

5. M. Henny, S. Oberholzer, C. Strunk and C. Schönenberger, "1/3shot- noise suppression in diffusive nanowires," Phys. Rev. B 59, 2871, (1999).

6. F. Liefrink, J. I. Dijkhuis, M. J. M. de Jong, L. W. Molenkamp, H. van Houten, "Experimental study of reduced shot noise in a diffusive mesoscopic conductor," Phys. Rev. B 49, 14066 (1994). 
7. P. Marconcini, M. Macucci, D. Logoteta, M. Totaro, "Is the regime with shot noise suppression by a factor $1 / 3$ achievable in semiconductor devices with mesoscopic dimensions?," Fluct. Noise Lett. 11, 1240012 (2012).

8. M. Macucci, P. Marconcini, "Effect of magnetic field on shot noise in diffusive conductors and cascaded barriers," J. Computational Electronics 7, 272 (2008).

9. P. Marconcini, M. Totaro, G. Basso, M. Macucci, "Effect of potential fluctuations on shot noise suppression in mesoscopic cavities," AIP Advances 3, 062131 (2013).

10. D. J. Thouless, S. Kirkpatrick, "Conductivity of the disordered linear chain', J. Phys. C 14, 235 (1981).

11. F. Sols, M. Macucci, U. Ravaioli and K. Hess, "Theory for a quantum modulated transistor," J. Appl. Phys. 66, 3892 (1989).

12. M. Macucci, A. T. Galick, and U. Ravaioli, "Quasi-threedimensional Green's-function simulation of coupled electron waveguides," Phys. Rev. B 52, 5210 (1995).

13. M. Macucci, L. Bonci, "Nanoscale device modeling," in "Handbook of Theoretical and Computational Nanotechnology," vol. 10, ed. M. Rieth and W. Schommers. American Scientific Publishers, Stevenson Ranch, CA, USA (2006).

14. M. Governale, D. Böse, "Magnetic barrier in confined twodimensional electron gases: Nanomagnetometers and magnetic switches,” Appl. Phys. Lett. 77, 3215 (2000).
15. P. Marconcini, M. Macucci, "Numerical techniques for the evaluation of conductance and noise in the presence of a perpendicular magnetic field," J. Computational Electronics 2, 387 (2003).

16. R. S. Whitney, P. Marconcini, M. Macucci, "Huge conductance peak caused by symmetry in double quantum dots," Phys. Rev. Lett. 102, 186802 (2009).

17. F. Guinea, J. A. Vergés, "Localization and topological disorder," Phys. Rev. B 35, 979 (1987).

18. E. N. Economou, "Green's functions in Quantum Physics." Springer-Verlag, Berlin (1990).

19. A. D. Stone, A. Szafer, "What is measured when you measure a resistance? - The Landauer formula revisited," IBM J. Res. Dev. 32, 384 (1988).

20. R. Landauer, "Spatial Variation of Currents and Fields Due to Localized Scatterers in Metallic Conduction," IBM J. Res. Dev. 1, 223 (1957).

21. M. Büttiker, "Scattering theory of thermal and excess noise in open conductors," Phys. Rev. Lett. 65, 2901 (1990).

22. J.-L. Pichard, "Random transfer matrix theory and conductance fluctuations," in "Quantum Coherence in Mesoscopic Systems," ed. B. Kramer, NATO ASI Series B 254, 369. Plenum, New York (1991).

23. S. Kettemann, R. Mazzarello, "Magnetolocalization in disordered quantum wires," Phys. Rev. B 65, 085318 (2002).

24. M. Büttiker, "Absence of backscattering in the quantum Hall effect in multiprobe conductors", Phys. Rev. B 38, 9375 (1988). 